\title{
Design of Dynamic Positioning Control System for an ROV with Unknown Dynamics Using Modified Time Delay Estimation
}

\author{
Alireza Hosseinnajad ${ }^{1}$, Mehdi Loueipour ${ }^{2 *}$ \\ ${ }^{1}$ Department of Mechanical Engineering, Isfahan University of Technology; alirezahosseinnajad@yahoo.com \\ ${ }^{2}$ Research Institute for Subsea Science and Technology, Isfahan University of Technology; loueipour@cc.iut.ac.ir
}

\section{ARTICLE INFO}

Article History:

Received: 31 Oct. 2018

Accepted: 27 Feb. 2019

\section{Keywords:}

Dynamic Positioning

Passive Arm

ROV

Time Delay Estimation

Unknown Dynamics

\begin{abstract}
In this paper, a control system is designed for dynamic positioning of an ROV with unknown dynamics, subject to external disturbances using passive arm measurements. To estimate uncertain dynamics and external disturbances, a new method based on time delay estimation (TDE) is proposed. The proposed TDE, not only maintains the advantages of conventional TDE, but also eliminates its sensitivity to sensor noise and fast-varying external disturbances which in turn, results in smooth control signal. The proposed control system is considered as a nonlinear PD-type controller together with feedforward of estimated dynamics and disturbances. This structure presents good performance against uncertainties and external disturbances which is guaranteed via stability analysis presented. To evaluate the performance of proposed TDE, simulations are conducted and comparison are made with conventional TDE. Besides, the performance of the proposed control system is compared with conventional time delay controller (TDC) and PID controller to verify its performance. Simulations show high accuracy and superior performance of the proposed control system.
\end{abstract}

\section{Introduction}

ROVs and AUVs play an important part in submarine operations such as inspection, equipment installation, scientific and military operation and exploration. To operate under harsh environmental conditions and maintain position and attitude, ROVs and AUVs are equipped with Dynamic positioning (DP) control systems not only to increase accuracy but also to decrease the work load on operators and result in increased safety factor [1].

Of the biggest challenges in dynamic positioning operations can the parametric uncertainties due to hydrodynamic effects, unknown external disturbances and accurate measurement of vehicle position be mentioned. Different control methods are considered in the literature to deal with these issues.

Application of robust controllers to deal with uncertainties and external disturbances has been considered for AUVs and ROVs in several works. Positioning of an ROV in horizontal plane was considered in [2] using sliding mode controller. Later, Healey and Lienard considered the application of multivariable sliding mode controller for controlling an AUV in steering and diving planes in [3]. In [4], a second order chattering free sliding mode controller was developed for positioning and trajectory tracking of an ROV. Control of an ROV in the horizontal plane using $H_{\infty}$ control method was considered in [5].

For plants with uncertainty, adaptive control is better compared to robust control methods in that the adaptation is performed with little or no bounds on the uncertainties [6]. However, adaptive control is applicable for adaptation of constant or slowly varying parameters. Also, linear parameterization of uncertainties is generally essential for deriving control laws. Different adaptive methods are utilized for positioning control of ROVs in horizontal plane and six degrees of freedom. In [7-8], adaptive and adaptive sliding mode controllers were designed for control of an ROV in horizontal plane. Experimental studies on ODIN ROV in six degrees of freedom are conducted in [9]. Comparison of adaptive controller with different control methods was conducted in [10]. Application of adaptive fuzzy sliding mode controller was considered in [11] and adaptive backstepping sliding mode controller was used in [12].

Application of online estimation techniques is another method to deal with uncertainties and external disturbances. Different methods are considered for online estimation of disturbances and unknown dynamics. In [13-17], extended state observers were considered to estimate unknown dynamics and external 
disturbances. Neural networks and fuzzy control systems are commonly used to estimate uncertainties and external disturbances in several works [18-20].

Another method to deal with uncertainties and external disturbances is the time-delay estimation (TDE). In time delay estimation, pervious system information, namely, vehicle acceleration and control signal are used to obtain estimation of unknown dynamics and external disturbances. The feedforward of estimated disturbances and unknown dynamics is considered to provide acceptable performance from control system. Application of time delay estimation was first considered for control of robotic manipulators in [21]. They combined TDE with sliding mode controller to reduce the gains of the discontinuous term.

The application of TDE for an underwater vehicle was considered in [6]. In this work, unknown dynamics were estimated by TDE and compensated for by a feedforward term introduced into a nonlinear PD-type controller. However, it was assumed that all system states are available. Experimental studies of TDEbased integral sliding mode controller on an AUV were considered in [22]. They employed Doppler Velocity Log (DVL) for obtaining AUV velocity. Moreover, Utilizing DVL in control system of the vehicle results in smaller sampling rates and increases the time delay. Besides, application of DVL is prone to integration drift and cannot be used in long-term applications such as DP, which require accurate and drift-free position signal of the vehicle.

For dynamic positioning operations, position and attitude of the ROV is required with high accuracy. To this effect, different measurement systems can be utilized in practice, among which, passive arm provides position and attitude with great accuracy, Figure 1. Passive arm can be considered as a robotic arm with passive joints. $P_{1}$ and $P_{2}$ are the connection points of the passive arm to ROV and underwater structure, respectively. Electromagnetic attachment system (EMAS) or vacuum cup may be used to attach passive arm to underwater structures. By measuring the joint angles with high accuracy, and through direct kinematics, one can obtain the position and orientation of the ROV with respect to point $P_{2}$. This would enable the ROV to have measurement of position and orientation at high sampling rates and accuracies and is free from drift.

In this paper, a new approach is proposed based on TDE which relies on passive arm measurements for DP of an ROV. The main feature of this approach is that time history of system dynamics is considered so that smooth estimate of unknown dynamics and external disturbances is obtained based on the mean value of dynamics over a short period of time. Furthermore, stability analysis of the proposed method is investigated. A simple but practical controller is utilized based on the estimated dynamics. The proposed structure entails the following advantages: i) significantly reduces the sensitivity of conventional TDE to sensor noise; ii) eliminates the sensitivity of conventional TDE against fast-varying disturbances; iii) provides smooth estimation of external disturbances and unknown dynamics which results in smoother control signal; iv) compared to PID-type controllers, which are designed in [23-26], it provides better positioning accuracies and control signal; v) increases the sampling rate so that smaller time delays can be achieved compared to sensors such as DVL.

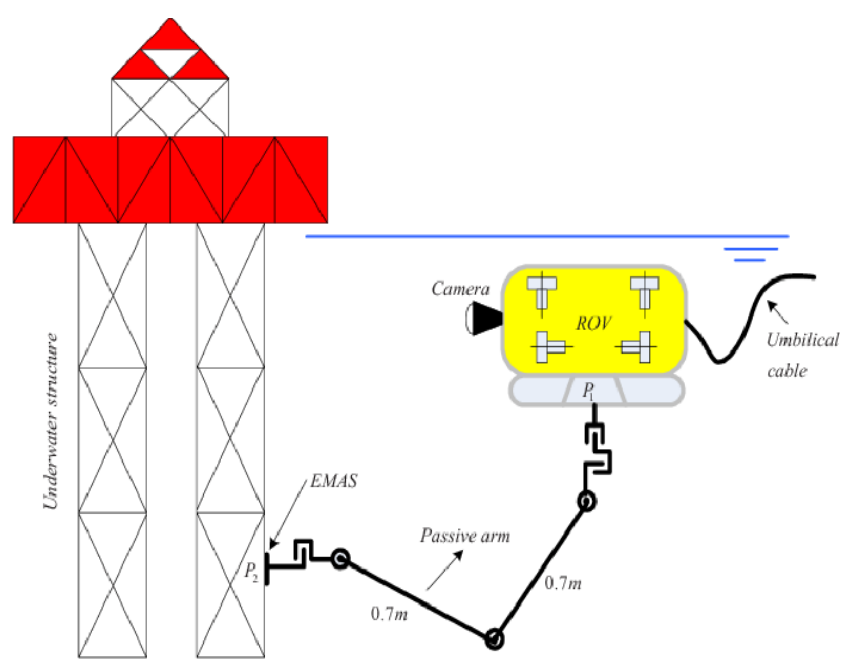

Figure 1. Dynamic positioning of an ROV with passive arm measurements [26]

\section{Governing Equations of ROV}

The governing equations of underwater vehicles are obtained with respect to two different coordinate systems, namely, inertial coordinate system, $\left\{x_{i}, y_{i}, z_{i}\right\}$, and body-fixed coordinate system, $\left\{x_{b}, y_{b}, z_{b}\right\}$, Figure 2, as

$$
\begin{aligned}
& \dot{\eta}=J(\eta) v \\
& M \dot{v}+C_{R B}(v) v+N\left(v_{r}\right) v_{r}+g(\eta)=\tau+d
\end{aligned}
$$

where $\eta=[x, y, z, \varphi, \theta, \psi]$ is the position and attitude vector in inertial coordinate system and $v=$ $[u, v, w, p, q, r]$ is the velocity vector in body-fixed frame. $v_{r}$ is the relative velocity of vehicle with respect to water which takes into account the velocity of ocean currents, $v_{\text {cur }}$, as $v_{r}=v-v_{\text {cur }} . M=M_{R B}+M_{A}$ is the inertia matrix including the added mass, $C_{R B}(v)$ is the rigid body Coriolis and centripetal matrix, $N\left(v_{r}\right)=$ $C_{A}\left(v_{r}\right)+D\left(v_{r}\right)$ which represents the effects of Coriolis and centripetal forces due to added mass and damping effects. $g(\eta)$ is the restoring forces due to weight and buoyancy, $\tau$ is the forces provided by controller and $d$ represents the external disturbances including the effects of passive arm. $J(\eta)$ is the transformation matrix between inertial and body-fixed frame defined as

$$
J(\eta)=\left[\begin{array}{cc}
J_{1} & 0 \\
0 & J_{2}
\end{array}\right]
$$

where 


$$
\begin{aligned}
& J_{1} \\
& =\left[\begin{array}{ccc}
c \psi c \varphi & -s \psi c \varphi+c \psi s \varphi s \theta & s \varphi s \psi+c \varphi s \theta c \psi \\
c \theta s \psi & c \varphi c \psi+s \varphi s \theta s \psi & -s \varphi c \psi+c \varphi s \theta s \psi \\
-s \theta & s \varphi c \theta & c \varphi c \theta
\end{array}\right] \\
& J_{2}=\left[\begin{array}{ccc}
1 & s \varphi t \theta & c \varphi t \theta \\
0 & c \varphi & -s \varphi \\
0 & s \varphi / c \theta & c \varphi / c \theta
\end{array}\right]
\end{aligned}
$$

where $s(),. c($.$) and t($.$) refer to \sin (),. \cos ($.$) and$ $\tan ($.$) , respectively.$

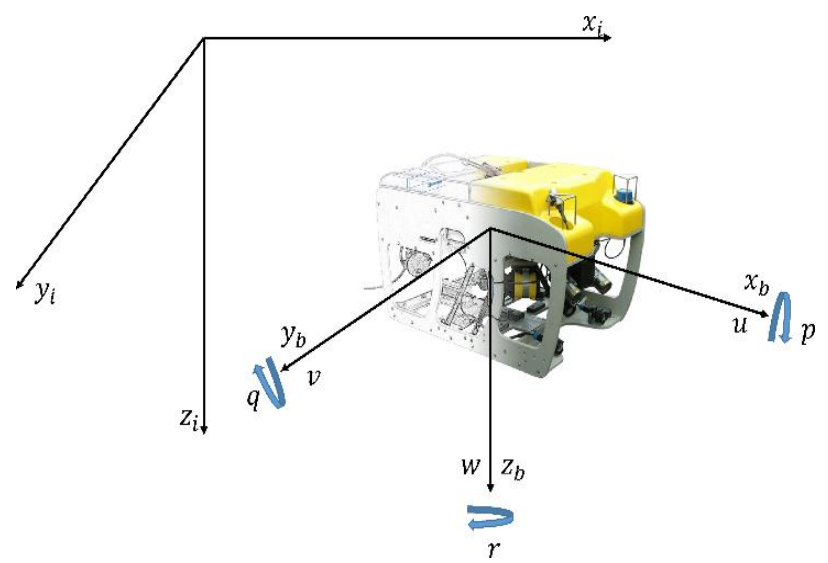

Figure 2. Inertial and body-fixed coordinate systems [1]

ROVs are designed such that roll and pitch angles are almost zero; By further simplification and assuming constant yaw angle $\left(\psi=\psi_{c}\right.$ and $\left.\dot{\psi} \approx 0\right)$, the vehicle motion can be considered in three degrees of freedom. The following sets of equations can be obtained for surge, sway and heave directions.

$$
\begin{aligned}
& \dot{\eta}=R\left(\psi_{c}\right) v \\
& \bar{M} \dot{v}=\tau+H
\end{aligned}
$$

where $\bar{M}$ is the nominal value of inertia matrix, $R\left(\psi_{c}\right)$ is the transformation matrix and $H$ is the unknown dynamics of the ROV, which are defined, respectively, as

$$
\begin{aligned}
& R=R\left(\psi_{c}\right)=\left[\begin{array}{ccc}
c \psi_{c} & -s \psi_{c} & 0 \\
s \psi_{c} & c \psi_{c} & 0 \\
0 & 0 & 1
\end{array}\right] \\
& H=\left[\begin{array}{c}
\left(m_{u}-\bar{m}_{u}\right) \dot{u}+k_{u} u+k_{u|u|} u|u|-d_{1} \\
\left(m_{v}-\bar{m}_{v}\right) \dot{v}+k_{v} v+k_{v|v|} v|v|-d_{2} \\
\left(m_{w}-\bar{m}_{w}\right) \dot{w}+k_{w} w+k_{w|w|} w|w|-d_{3}
\end{array}\right]
\end{aligned}
$$

where $m_{u}, m_{v}$ and $m_{w}$ are ROV masses including the added mass, $\bar{m}_{u}, \bar{m}_{v}$ and $\bar{m}_{w}$ are the corresponding nominal values and $k_{u}, k_{v}$ and $k_{w}$ are linear damping coefficients along the $x, y$ and $z$ axes, respectively. $k_{u|u|}, k_{v|v|}$ and $k_{w|w|}$ represent nonlinear damping terms along the respective axes and $d_{1}, d_{2}$ and $d_{3}$ are the unmodeled dynamics and external disturbances.

\section{Proposed Modified Time Delay Control and Estimation}

The block diagram of the control system is shown in Figure 3. The control objective is to drive the ROV to the desired position in the presence of unknown dynamics and external disturbance. To estimate unknown dynamics of the ROV, i.e. $H$, consider the second equation of (4) as

$$
H=\bar{M} \dot{v}-\tau
$$

By introducing a small time delay into $\dot{v}$ and $\tau$, one may obtain the following relation for estimation of $H$ as

$$
H \approx \widehat{H}=\bar{M} \dot{v}_{(t-\delta)}-\tau_{(t-\delta)}
$$

where $\delta$ is the time delay introduced into the control law and acceleration to obtain $\widehat{H}$.

This method, TDE, is simple in that, it only requires the introduction of time delay into system dynamics to obtain estimate of external disturbances and designing other structures such as disturbance observers or the introduction of integral term into the controller is not required. However, TDE leads to significant sensitivity of the control signal to sensor noise because of numerical differentiation for calculating acceleration of the vehicle from

$$
\dot{v}_{(t-\delta)}=R^{-1} \frac{\left(\eta_{t}-2 \eta_{(t-\delta)}+\eta_{(t-2 \delta)}\right)}{\delta^{2}}
$$

This effect is intensified when the sampling rate is high, since for lower $\delta$, the sensitivity to sensor noise increases significantly. This may lead to instability of the vehicle and mechanical wear and tear of the thrusters. However, application of lower sampling rates will lead to large time delays and deteriorate the performance of control system.

To deal with this problem, so that high sampling rates are maintained and significant reduction of sensitivity to sensor noise is achieved, the following method is proposed for estimating $H$. In this method, instead of estimating $H$ with a single set of data, a series of previous data are used as

$$
\widehat{H}_{m}=\frac{\sum_{p=1}^{n}\left(\bar{M} \dot{v}_{(t-p \delta)}-\tau_{(t-p \delta)}\right)}{n}
$$

where $n$ is the total number of data considered in the time period $n \delta$. This modification, due to high sampling rate of passive arm and high bandwidth of the vehicle, will maintain the advantages of conventional TDE provided that $\delta$ is sufficiently small. In practice $\delta$ is considered as the sampling rate which is in the range $0.01-0.005 s$ for passive arm [6, 24].

The proposed control law, based on modified TDE, can be defined as 


$$
\begin{aligned}
\tau=\bar{M} R^{-1}\left(\ddot{\eta}_{d}-\right. & k_{p}\left(\eta-\eta_{d}\right) \\
& \left.-k_{d}\left(\dot{\eta}-\dot{\eta}_{d}\right)\right)-\widehat{H}_{m}
\end{aligned}
$$

where $\eta_{d}$ and $\dot{\eta}_{d}$ are the desired values of $\eta$ and $\dot{\eta}$, respectively, and $\ddot{\eta}_{d}$ is the desired acceleration. $k_{p}$ and $k_{d}$ are positive diagonal gain matrices of appropriate dimensions.

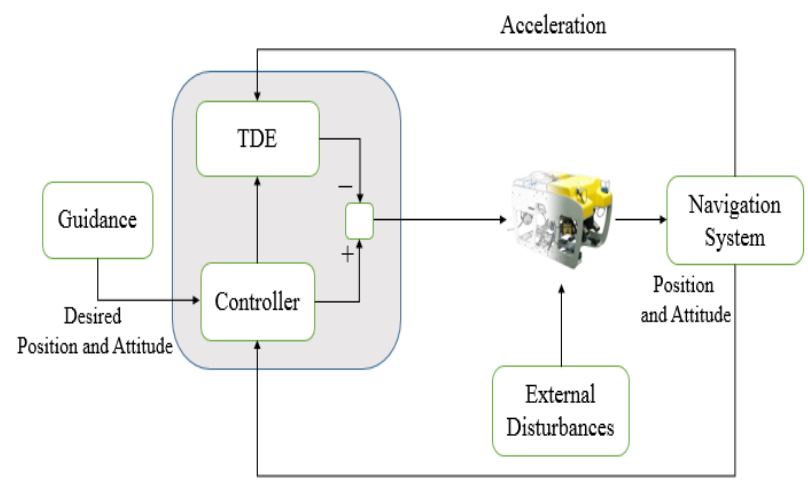

Figure 3. Block-diagram of control system

\section{Stability Analysis}

Consider the system (4), the controller (11) and the estimation (10). Transforming Eq. (4) into inertial coordinate system may result in

$$
\begin{aligned}
& \bar{M} R^{-1} \ddot{\eta} \\
& =\bar{M} R^{-1}\left(\ddot{\eta}_{d}-k_{p}\left(\eta-\eta_{d}\right)-k_{d}\left(\dot{\eta}-\dot{\eta}_{d}\right)\right) \\
& \quad+\left(H-\widehat{H}_{m}\right)
\end{aligned}
$$

which shows that the error dynamics is obtained as

$$
\ddot{e}+k_{d} \dot{e}+k_{p} e=R \bar{M}^{-1}\left(H-\widehat{H}_{m}\right)
$$

The term $R \bar{M}^{-1}\left(H-\widehat{H}_{m}\right)$ can be formulated as

$$
\begin{aligned}
& R \bar{M}^{-1}\left(H-\widehat{H}_{m}\right) \\
& \quad=R \bar{M}^{-1} \frac{\sum_{p=1}^{n} n H(t)-H(t-p \delta)}{n}
\end{aligned}
$$

It can be considered that

$$
H(t)-H(t-p \delta)=\varepsilon_{p}(t)
$$

where $\varepsilon_{p}(t)$ is bounded for small $\delta$ and converges to zero as $\delta \rightarrow 0$, provided that $\bar{M}$ is an acceptable estimate of $M$ [6]. Consequently,

$$
\varepsilon(t)=R \bar{M}^{-1} \frac{\sum_{p=1}^{n} \varepsilon_{p}(t)}{n}
$$

which is bounded because of boundedness of each $\varepsilon_{p}(t)$. Therefore, the error dynamics can be formulated as

$$
\ddot{e}+k_{d} \dot{e}+k_{p} e=\varepsilon(t)
$$

which results in input to state stability, bounded-input bounded-output stability and global uniform ultimate boundedness of closed-loop error dynamics for timevarying disturbances and global exponential stability of error dynamics for constant or very slowly-varying dynamics.

\section{Simulation Studies}

Simulations are carried out in two different cases to verify the performance of modified TDE and proposed control system. First, the modified TDE is compared with conventional TDE of [6] in terms of estimation accuracy and sensitivity to sensor noise. Later, the performance of the proposed control system is compared with the structure of [6] and PID controller. All the simulations are conducted in MATLAB/ Simulink.

The controller parameters are taken as $k_{p}=$ $\operatorname{diag}(5,2.3,5.1), k_{p}=\operatorname{diag}(12,7.2,13.2)$ and $\bar{M}=$ $\operatorname{diag}(300,500,500)$. The parameters of the ROV are given in Table 1 as

Table 1. Parameters of ROV

\begin{tabular}{ccc}
\hline$m_{u}(\mathrm{~kg})$ & $m_{v}(\mathrm{~kg})$ & $m_{w}(\mathrm{~kg})$ \\
\hline 391.5 & 639.6 & 639.6 \\
\hline$k_{u}$ & $k_{v}$ & $k_{w}$ \\
\hline 16 & 131.8 & 65.6 \\
\hline$k_{u|u|}$ & $k_{v|v|}$ & $k_{v|v|}$ \\
\hline 229.4 & 328.3 & 296.8 \\
\hline
\end{tabular}

The disturbances are considered as follows.

- For the first 60 seconds, there is no external disturbance.

- After 60 seconds, external disturbances are considered based on Wiener process [27]. This model for each degree of freedom is defined as

$$
\dot{d}=E_{d} w
$$

where $E_{d}$ is the amplitude of the Gaussian white noise $w$. For simulation, it is considered that $E_{d}=300 I$.

- In sway direction, to model wave-induced motions, a sinusoidal disturbance of amplitude $200 \mathrm{~N}$ and frequency of $\pi / 5(\mathrm{rad} / \mathrm{s})$ is considered from time $60 \mathrm{~s}$.

The time delay is taken $\delta=0.01 s$ and the number of previous data are taken as $n=10$.

\subsection{Time Delay Estimation}

First, the performance of the proposed TDE is considered in estimating disturbances and unknown dynamics and the results of the simulations are presented in Figure 4. To evaluate the sensitivity of both methods to measurement noise, position measurement noise is considered. For this, a bandlimited white noise is considered with noise power of $10^{-10}$.

Figures $4 \mathrm{a}-4 \mathrm{c}$ show that the proposed TDE has estimated unknown dynamics and external disturbance 
(a)

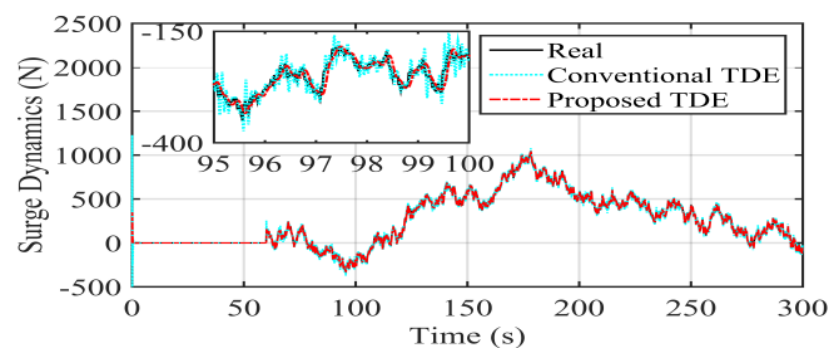

(b)

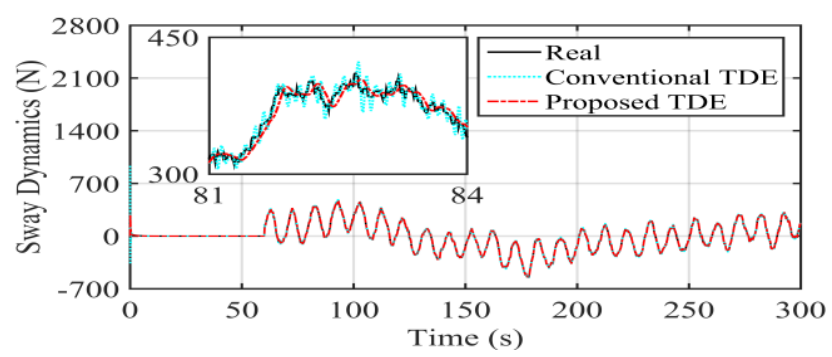

(c)

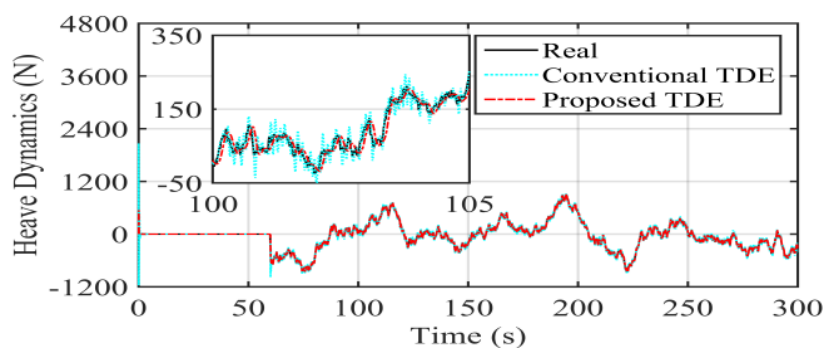

(d)

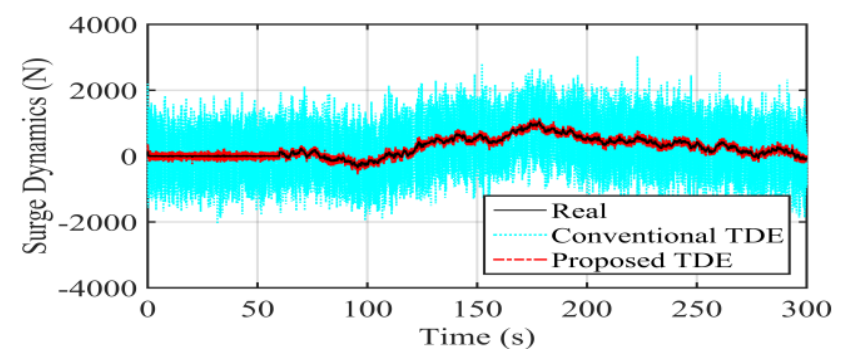

(e)

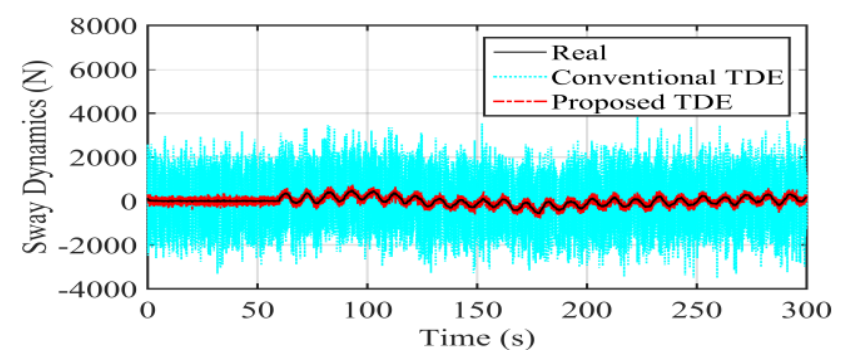

(f)

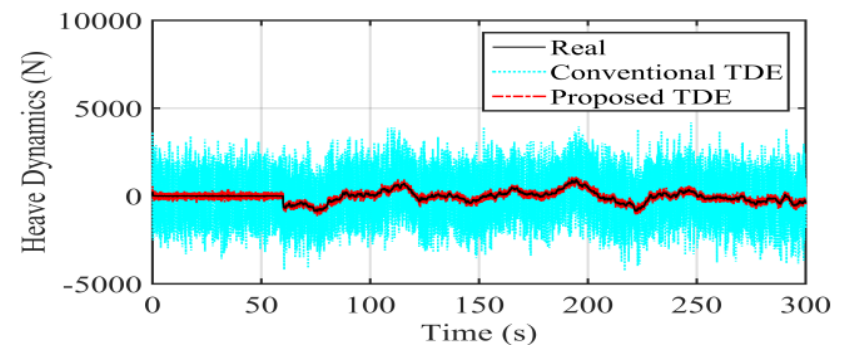

Figure 4. Simulation result for comparing conventional TDE and proposed TDE for estimation of ROV dynamic for corresponding degrees of freedom; (a)-(c): Without position sensor noise, (d)-(f): With position sensor noise effects.

with high accuracy and a smooth signal is obtained for all degrees of freedom compared to conventional TDE. The smoothness of the proposed TDE relies on the averaging method taken into consideration. In terms of sensor noise effect, Figure 4d-4f, conventional TDE has led to signification oscillations of random nature. These oscillations, when considered in closed-loop control system, lead to significant oscillations in control signal which is quite inapplicable. However, the proposed TDE has estimated unknown dynamics quite well and the effect of sensor noise is not so much evident.

\subsection{Control System}

The performance of control systems is presented in term of position and control signal for different degrees of freedom in Figure 5.

The performance of controllers in terms of position signal shows that the proposed control system and the structure of [6] has maintained the position of ROV at desired values within an accuracy of a couple of millimeters in all degrees of freedoms. The control systems based on TDE has led to better results compared to PID controller. The application of PID controller, leads to significant overshoot in transients and has resulted in significant errors in steady-state part. The superior performance of TDE based controllers is outlined in sway direction where the position of ROV in maintained with excellent accuracy, whereas, PID controller has led to oscillations of high amplitude.

The proposed control system, despite providing similar control signal to PID controller, has led to better positioning accuracies which is due to estimation of unknown dynamics and compensation by a feedforward term. It is as if the disturbances are compensated for prior to causing deviations from desired position, whereas, PID controller is based on error growth and compensates for external disturbances after the error is introduced into system. This feature makes the proposed control system more fastresponding than PID controller. This effect is evident when ROV is subjected to external disturbances at time $60 \mathrm{~s}$. The proposed controller has eliminated the effects of external disturbances and resulted in better accuracies compared to PID controller, which shows that the proposed controller responds faster to external disturbances.

The application of integral term in PID controller has led to high level of control effort in transients which is 2-3 times more than the control signal of proposed control system. Besides, the control signal of structure of [6] is similar to control signal of PID controller in transients and leads to high level of control effort, 
whereas the proposed structure leads to smooth estimation in transients and decreases the control effort. In terms of sensor noise effect in control signal, Figures 5d-5f, PID controller and proposed structure have provided control signals less sensitive to sensor noise, while, significant variations are evident in control signal provided by structure of [6]. It is also evident that the proposed control system has provided better control signal compared to PID controller.

\section{Conclusion}

In this paper, a modified time delay estimation based control system was introduced for precise positioning of an ROV with unknown dynamics and subject to external disturbances using passive arm measurements. The modification introduced into conventional time delay estimation made it less sensitive to measurement noise and provided smooth estimates of fast-varying external disturbances. The performance of the proposed TDE was also evaluated under sensor noise.

(a)

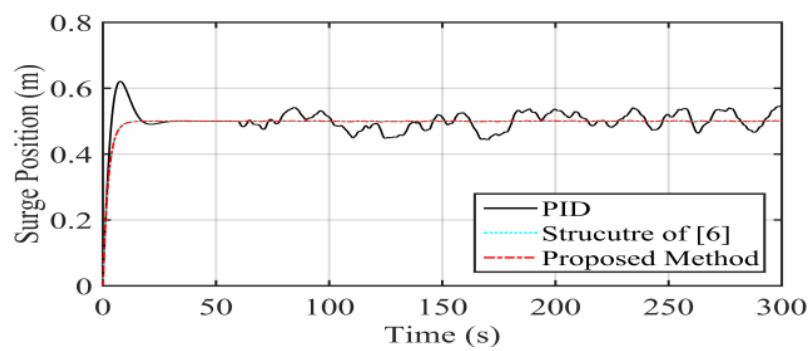

(b)

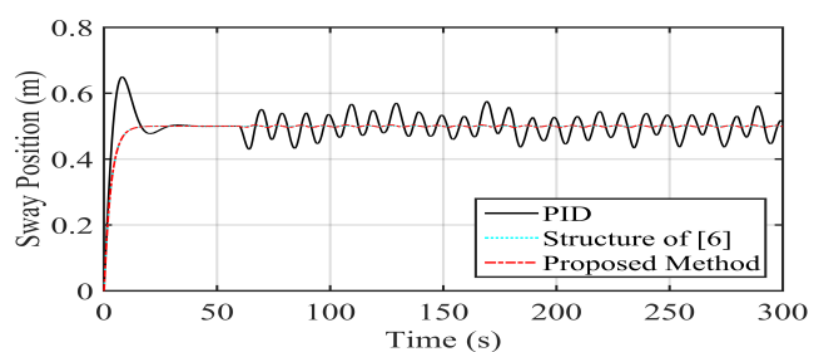

(c)

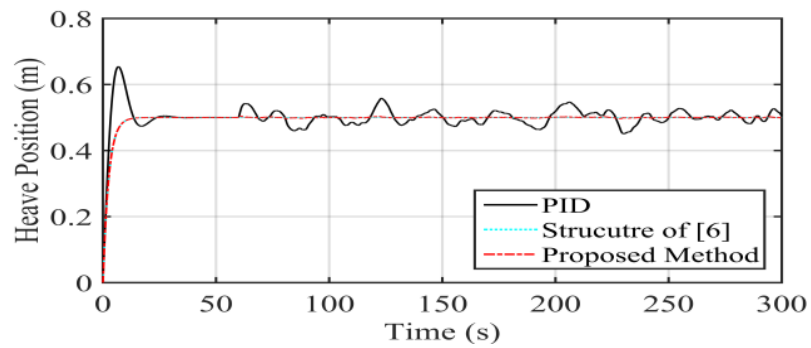

It was surprisingly witnessed that sensor noise effect was significantly reduced both in estimation and control signals. To evaluate the performance of proposed control system, simulations were conducted and the results showed that the proposed control system has maintained the accuracy of conventional method while removing its sensitivity to sensor noise and fast varying disturbances, which in turn, results in smoother control signal applicable by thruster system. Besides, the proposed control system improved the control signal in transients compared to conventional method and PID controller which the former relies on conventional TDE and the latter depends on integral action. The stability analysis was also presented for error dynamics of the closed-loop control system and it was shown that the error dynamics is GUUB for fastvarying disturbances and GES for constant or very slowly-varying disturbances.

(d)

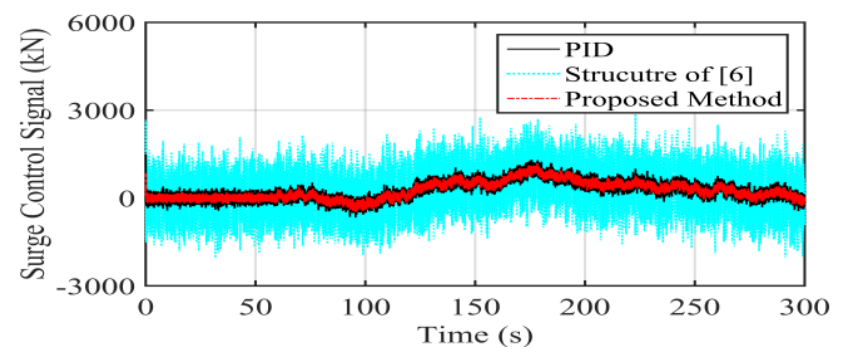

(e)

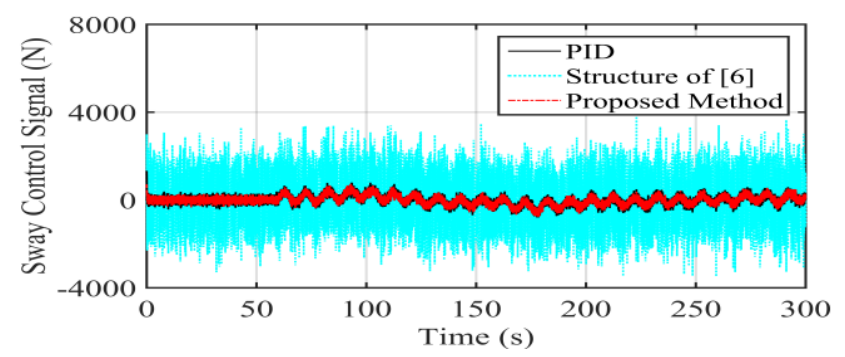

(f)

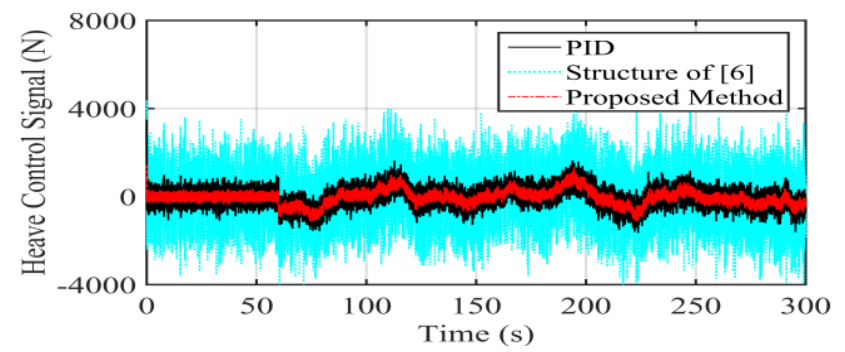

Figure 5. Simulation result for comparing the performance of different control methods in the presence of position sensor noise; (a)-(c): Position, (d)-(f): Control signal.

\section{References}

1- Hosseinnajad, A. and Loueipour, M., (2018), Dynamic Positioning of an ROV with Unknown Dynamics and in the Presence of External Disturbances Using Extended-State Observer, $20^{\text {th }}$ Marine Industries Conference, Tehran, Iran.
2- $\quad$ Yoerger, D. R. and Slotine, J. J. E., (1985), Robust Trajectory Control of Underwater Vehicles, IEEE Journal of Oceanic Engineering, Vol. 10(4), p. 462-470.

3- Healey, A. J. and Lienhard, D., (1993), Multivariable sliding mode control for autonomous diving and steering of unmanned underwater vehicles, 
IEEE Journal of Oceanic Engineering, Vol. 18(3), p. 327-339.

4- Valdovinos, L. G. G., Jimenez, T. S. and Rodriguez, H. T., (2009), Model free high order sliding mode control for ROV: station keeping approach, OCEANS, Biloxi, USA.

5- $\quad$ Conte, G. and Serrani, A., (1998), Robust control of a remotely operated underwater vehicle, Automatica, Vol. 34(3), p. 193-198.

6- Kumar, R. P., Dasgupta, A. and Kumar, C. S., (2006), Robust trajectory control of underwater vehicles using time delay control law, Ocean Engineering, Vol. 34, p. 842-849.

7- Slotine, J. J. E. and Benedetto, M. D. D., (1990), Hamiltonian adaptive control of spacecraft, IEEE Transactions on Automatic Control, vol. 35(7), p. 848-852.

8- Fossen, T. I. and Sagatun, S. I., (1991), Adaptive control of nonlinear underwater robotic systems, Proceedings of the IEEE International Conference on Robotics and Automation, Sacramento, California.

9- $\quad$ G. Antonelli, S. Chiaverini, N. Sarkar, and M. West, "Adaptive control of autonomous underwater vehicle: Experimental results on ODIN," IEEE Transactions on Control Systems Technology, vol. 9, no. 5, pp. 756-765, 2001.

10- Smallwood, D. A. and Whitcomb, L., (2004), Model-based dynamic positioning of underwater robotic vehicles: theory and experiments, IEEE Journal of Oceanic Engineering, Vol. 29(1), p. 169-186.

11- Mohammad, A. R., Eghtesad, M. and Kamali, R., (2011), A robust adaptive fuzzy sliding mode controller for trajectory tracking of ROVs, IEEE Conference on Decision and Control and European Control Conference, Orlando, USA.

12- Patompak, P. and Nikhamhang, I., (2012), Adaptive backstepping sliding mode controller with bound estimation for underwater robotic vehicles, International Conference on Electrical Engineering/ Electronics, Computer, Telecommunications and Information Technology, Thailand.

13- Liu, Y., Kung, T., Chang, K. and Chen, S., (2013), Observer-based adaptive sliding mode control for pneumatic servo system, Precision Engineering, Vol. 37, p. 522-530.

14- Yao, J., Jiao, Z., and Ma, D., (2014), Extendedstate-observer-based output feedback nonlinear robust control of hydraulic systems with backstepping, IEEE Transactions on Industrial Electronics, Vol. 61(11), p. 6285-6293.

15- Qian, J., Xiong, A., and Ma, W., (2016), Extended state observer-based sliding mode control with new reaching law for PMSM speed control, Mathematical Problems in Engineering, Vol. 2016, https://doi.org/10.1155/2016/6058981.
16- Castaneda, H., Salas-Pena, O., and LeonMorales, J., (2017), Extended observer based on adaptive second order sliding mode control for a fixed wing UAV, ISA Transactions, Vol. 66, p. 226-232.

17- Cui, R., Chen, L., Yang, C., and Chen, M., (2017), extended state observer-based integral sliding mode control for an underwater robot with unknown disturbances and uncertain nonlinearities" IEEE Transactions on Industrial Electronics, Vol. 64(8), p. 6785-6795.

18- $\quad$ Tong, S., Wang, T., Li, Y., and Zhang, H., (2014), Adaptive neural network output feedback control for stochastic nonlinear systems with unknown dead-zone and unkodeled dynamics, IEEE Transactions on Cybernetics, Vol. 44(4), p. 910-921.

19- $\quad \mathrm{Hu}, \mathrm{X} ., \mathrm{Du}, \mathrm{J}$. , and Shi, J., (2015), Adaptive fuzzy controller design for dynamic positioning system of vessels, Applied Ocean Research, Vol. 53, p.46-53.

20- He, W., Huang, H., and Ge, S. S., (2017), Adaptive neural network control of a robotic manipulator with time-varying output constraints, IEEE Transactions on Cybernetics, Vol. 47(10), p. $3136-3147$.

21- Elmali, H., and Olgac, N., (1992), Theory and implementation of sliding mode control with perturbation estimation (SMCPE), IEEE International Conference on Robotics and Automation, Nice, France. 22- Kim, J., Joe, H., Yu, S., Lee, J. S., and Kim, M., (2016), Time delay controller design for position aly.control of autonomous underwater vehicles under disturbances, IEEE Transactions on Industrial Electronics, p. 1-10, DOI: 10.1109/TIE.2015.2477270. 23- Hsu, L., Costa, R. R., Lizarralde, F., and Cunha, J. P., (1999), Passive Arm based dynamic positioning system for remotely operated underwater vehicle, Proceedings of the IEEE international Conference on Robotics and Automation, Michigan, USA.

24- Hsu, L., Costa, R. R., Lizarralde, F., and Cunha, J. P., (2000), Dynamic positioning of remotely operated underwater vehicles, IEEE Robotics and Automation Magazine, Vol. 7(3), p. 21-31.

25- Hoang, N. Q., and Kreuzer, E., (2006), adaptive PD-controller for positioning of a remotely operated vehicle close to an underwater structure: theory and experiments, Control Engineering Practice, Vol. 15, p. 411-419.

26- Lei, Q., Lixing, Z., and Weidong, Z., (2016), robust adaptive PID control for positioning of remotely operated vehicles working in close proximity of underwater structures, $35^{\text {th }}$ Chinese Control Conference.

27- Candeloro, M., Sorensen, A. J., Longhi, S., and Dukan, F., (2012), Observers for dynamic positioning of ROVs with Experimental results, IFAC Conference on Maneuvering and Control of Marine Craft, Arenzano, Italy 